\title{
Sleep Paralysis: An Overlooked Phenomenon
}

\author{
Ambreen Kalam, Fazal Ameen Habib
}

Dear Sir,

'There is a time for many words, and there is also a time for sleep'Homer, the Odyssey. A recent systemic review of an aggregated data from 35 empirical studies $(\mathrm{N}=36,533$ subjects) found that $7.6 \%$ of the general population experienced at least one Sleep paralysis episode over the course of their life. Higher lifetime prevalence rates were found in students $(28.3 \%)$ and psychiatric patients $(31.9 \%)^{1}$.

Sleep paralysis is a commonly attributed state characterized by the inability to move either at the onset of sleep or upon awakening accompanied by hypnagogic experiences which are visual, auditory and sensory hallucinations ${ }^{2}$. It can occur as an isolated, familial or tetrad of narcolepsy. If it occurs while you are falling asleep, it is called hypnagogic or predormital sleep paralysis. If it happens as you are waking up, its called hypnopompic or postdormital sleep paralysis. Sleep paralysis has been estimated to effect approximately $1.7 \%$ to $40 \%$ of general population with victims predominantly students ${ }^{3}$. It typically peaks at the thirties and appears to be associated with post-traumatic stress disorder (PTSD), narcolepsy and panic attacks. Other major contributing factors include sleep deprivation, fatigue and stress. Alarming symptoms of Sleep Paralysis include feeling paralyzed, hear deafening buzzing sounds, feel electrical sensations shooting throughout the body. Difficulty in breathing due to an oppressive weight on the chest and body and a sense of suffocation (Adler). Person may hear footsteps or see indistinct shapes approaching them.

This condition is representing an upward trend for those who have frenetic and tedious jobs, because of hectic working hours, such individuals tend to get disturbed sleep and usually depend on caffeinated drinks to stay awake which all contribute in the long run to this condition. These symptoms need urgent treatment if they are disturbing the daily activities of a before normal healthy person. To start with, lifestyle modification tops the list which includes:

- Improving sleep hygiene and minimizing sleep deprivation

- Avoiding drugs and alcohol

- Sleeping on the side instead of the back

- Avoiding stimulants like caffeine and large meals before bed

- Minimizing exposure to blue light before bed

- Treating any mental health problems such as bipolar disorder or narcolepsy or leg cramps.

- Using antidepressant medications if prescribed by physician $^{5}$

Hence it is imperative to note that sleep paralysis is a sleeping disorder which is drastically gaining momentum due to increase in stressful jobs and towering amount of competition which is very frequent in every field. To avoid this, a positive and healthy working environment must be enforced, along with sufficient time for family life. "A well-spent day brings happy sleep”, Leonardo da Vinc.

\section{REFERENCES:}

1. Sharpless BA, Barber JP. Lifetime prevalence rates of sleep paralysis: a systematic review. Sleep Med Rev. 2011;15(5):311-315

2. http://www.sleepeducation.org/sleepdisorders-bycategory/parasomniacs/sleepparalysis/overview-facts

3. Cheyne JA. Sleep paralysis episode frequency and number, types and structure of associated hallucinations. J sleep Res.2005;14:319-24

4. https://www.researchgate.net/publication/6352167

5. https://www.webmd.comsleep-disorders/guide/sleep-paralysis

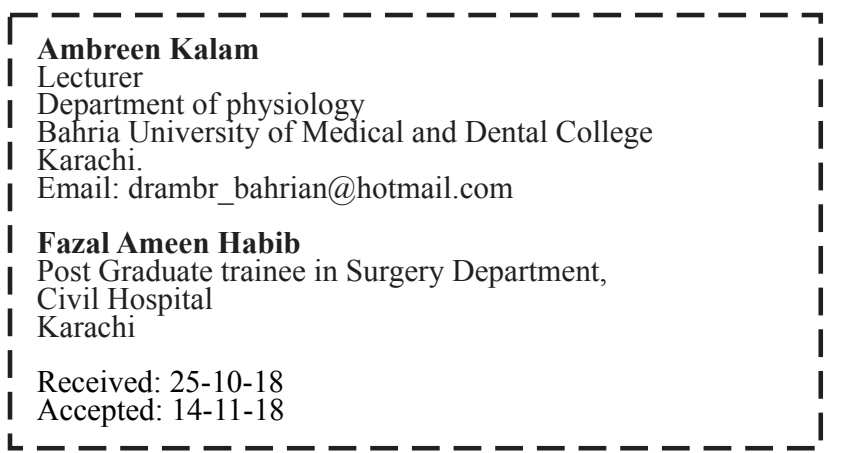

